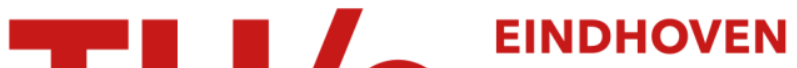 UNIVERSITY OF TECHNOLOGY
}

\section{MIMO jerk derivative feedforward for motion systems}

Citation for published version (APA):

Boerlage, M. L. G. (2006). MIMO jerk derivative feedforward for motion systems. In Proceedings of the 2006 American Control Conference (ACC 2006), June 14-16, 2006, Minneapolis, Minnesota, USA Institute of Electrical and Electronics Engineers.

Document status and date:

Published: 01/01/2006

\section{Document Version:}

Accepted manuscript including changes made at the peer-review stage

\section{Please check the document version of this publication:}

- A submitted manuscript is the version of the article upon submission and before peer-review. There can be important differences between the submitted version and the official published version of record. People interested in the research are advised to contact the author for the final version of the publication, or visit the $\mathrm{DOI}$ to the publisher's website.

- The final author version and the galley proof are versions of the publication after peer review.

- The final published version features the final layout of the paper including the volume, issue and page numbers.

Link to publication

\section{General rights}

Copyright and moral rights for the publications made accessible in the public portal are retained by the authors and/or other copyright owners and it is a condition of accessing publications that users recognise and abide by the legal requirements associated with these rights.

- Users may download and print one copy of any publication from the public portal for the purpose of private study or research.

- You may not further distribute the material or use it for any profit-making activity or commercial gain

- You may freely distribute the URL identifying the publication in the public portal.

If the publication is distributed under the terms of Article 25fa of the Dutch Copyright Act, indicated by the "Taverne" license above, please follow below link for the End User Agreement:

www.tue.nl/taverne

Take down policy

If you believe that this document breaches copyright please contact us at:

openaccess@tue.nl

providing details and we will investigate your claim. 


\title{
MIMO jerk derivative feedforward for motion systems
}

\author{
Matthijs Boerlage
}

\begin{abstract}
This paper shows that flexible modes in motion systems result in residual dynamics that can not be reduced using conventional acceleration feedforward and static decoupling. When reference trajectories with low frequency excitation are applied, low frequency tracking errors and cross-talk errors occur as peaks during jerk phases of a motion. A multivariable jerk derivative feedforward controller is presented which compensates for the joint contribution of all flexible modes in the low frequency region. Furthermore it is shown that no higher order (than 4) feedforward controller is required to improve low frequency tracking performance. A simulation example of a positioning device shows a significant improvement of the tracking performance.
\end{abstract}

\section{INTRODUCTION}

Industrial motion control systems require high tracking performance involving short move times and small settling times. Typical applications are positioning devices in semiconductor industry, DVD player mechanisms and many robots. In most of these applications, a combination of feedforward and feedback control is used in a two degree of freedom control architecture, see Figure 1. Herein, the

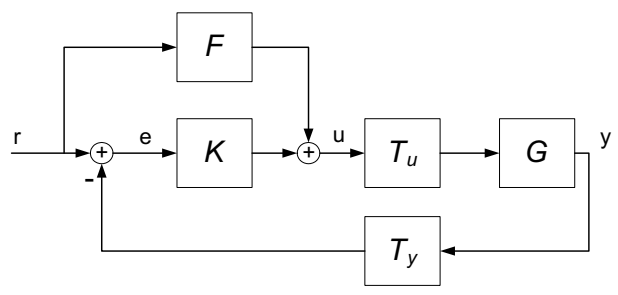

Fig. 1. Multivariable control architecture used in this work.

signals $r, e, u, y$ are the reference trajectory, servo error, plant input and plant output. The systems, $G, K, F$ denote the plant, feedback controller and feedforward controller respectively. The design of the feedback controller is not discussed in this work, however we focus on the situation that a decentralized controller will be used, [12]. Hence, static input and output decoupling transformations $T_{u}, T_{y}$ are facilitated to achieve the required level of diagonal dominance, [7].

As $T_{u}, T_{y}$ do not vary per frequency, decoupling of the rigid body dynamics does not necessarily imply decoupling of the flexible dynamics. When smooth step and scanning motions are applied, mostly low frequency

M. Boerlage is with the Control Systems Technology group of the Faculty of Mechanical Engineering, Technische Universiteit Eindhoven, $5600 \mathrm{MB}$ Eindhoven, The Netherlands m.l.g.boerlagedtue.nl dynamics are excited. It will be shown that this low frequency tracking problem requires a different approach than that of residual vibration suppression as discussed in [9] and references therein.

The contribution of this work is a multivariable extension of [2] which not only improves tracking performance of the diagonal terms, but also reduces cross-talk significantly. Therefore, design freedom which is not exploited due to static decoupling can be recovered using this multivariable feedforward controller.

The outline of this paper is as follows. The following section discusses the properties of the reference profiles used in the applications we study. Next, properties of the plant are discussed, followed by a section on the implication of static decoupling techniques. Subsequently, an analysis of low frequency tracking performance is presented from which the multivariable feedforward controller is proposed in Section VI. Finally a simulation example is presented to illustrate the newly developed theory.

\section{REFERENCE PROFILES}

Many industrial motion systems are designed to perform step and scanning movements. Typically, piecewise finite order polynomials are used as reference profile. These profiles have motion phases with constant velocity, acceleration, jerk, the derivative of jerk, etc. Reference trajectories are designed to contain mostly low frequency energy, hence resonance dynamics are little excited. If this would not be the case, excitation of resonance dynamics should be avoided using methods known as input shaping techniques, see [5],[6],[9] and references therein. Throughout this work, it is assumed that the fourth derivative of the position trajectory exists, in [2] it was shown that due to digital implementation this requirement is often met in practice.

\section{Plant DYNAMics}

We focus on linear time invariant electromechanical systems which have the same number of actuators and sensors as rigid body modes. These systems are typically constructed to be light and stiff, so that resonance modes appear far above the bandwidth of the feedback controller. As the mechanical dynamics are dominant, one can assume that these systems have low internal damping which is considered being proportional. Also it is assumed that friction or damping to the world can be neglected or is compensated for otherwise. The 
plant can then be expressed as;

$$
\begin{aligned}
G(s) & =\underbrace{\sum_{j=1}^{N_{r b}} \frac{u_{j}^{T} v_{j}}{s^{2}}}_{\text {rigid dynamics }}+\underbrace{\sum_{i=N_{r b}+1}^{N} \frac{u_{i}^{T} v_{i}}{\left(s^{2}+2 \zeta_{i} \omega_{i} s+\omega_{i}^{2}\right)}}_{\text {flexible dynamics }} \\
& =G_{r b} \frac{1}{s^{2}}+G_{\text {flex }}(s)
\end{aligned}
$$

where $\zeta_{i}, \omega_{i}$ are the relative damping and the resonance frequencies of the flexible modes respectively. The eigenmodes of the plant are denoted by $u$ and $v$. Note that at $s=0$, the contribution of the flexible part of the dynamics is a constant which we define as $\hat{G}_{\text {flex }}=G_{\text {flex }}(0)$. Hence, when a simplified model of the plant is made in the frequency region below the first resonance, the model contains a constant matrix representing all modal contributions in low frequencies added to a rigid body model, so that

$$
\hat{G}(s)=G_{r b} \frac{1}{s^{2}}+\hat{G}_{f l e x}
$$

This model equals the singular perturbation approximation of the plant dynamics, see [11].

\section{Decoupling}

In industrial practice, decentralized controllers are used to allow design based on single input single output loopshaping theory. To facilitate independent design of the elements of the decentralized feedback controller, diagonal dominance is required, [3], [7]. A system is diagonal dominant when

$$
\begin{array}{cl}
\rho\left(G_{i i}(s)^{-1} G_{i j}(s)\right)<1 & \forall s \in \mathcal{D}_{N}, \\
G(s)=G_{i i}(s)+G_{i j}(s), & \operatorname{det}\left(G_{i i}(s)\right) \neq 0
\end{array}
$$

were $\rho($.$) is the spectral radius, \mathcal{D}_{N}$ is the Nyquist contour while $G_{i i}(s)$ and $G_{i j}(s), i \neq j$ are the diagonal and nondiagonal elements of $G(s)$ respectively. In order to achieve diagonal dominance, static input and output transformations $T_{u}, T_{y}$ are used to decouple the rigid body dynamics. Decoupling of the rigid body dynamics expresses the servo errors in the cartesian framework which is favorable for practical control design. The transformations $T_{u}, T_{y}$ can be derived considering the kinematics of the plant, [1], or considering frequency response functions, [10].

When the rigid body decoupling input and output transformations are applied, we can define the plant as

$$
\begin{aligned}
\tilde{G}(s) & =T_{y} \hat{G}(s) T_{u}=T_{y} G_{r b} T_{u} \frac{1}{s^{2}}+T_{y} \hat{G}_{f l e x} T_{u} \\
& =\tilde{G}_{r b} \frac{1}{s^{2}}+\tilde{G}_{f l e x}
\end{aligned}
$$

where now $\tilde{G}_{r b}$ is diagonal while the term $\tilde{G}_{f l e x}$ can still exhibit interaction. In many practical situations, interaction of the flexible modes might increase slightly when the rigid body modes are decoupled. This is because, in many cases, the eigenmodes of the flexible dynamics do not have the same alignment as the eigenmodes of the rigid body dynamics. Due to the term $\frac{1}{s^{2}}$, the system is diagonal dominant in low and midrange frequencies and as long as Equation 3 is valid, a small amount of interaction is still allowed. However, from a performance point of view, any residual interaction means crosstalk so it may still be necessary to compensate for this.

In order to eliminate crosstalk, it is desirable to decouple the path from the disturbances to the servo error. For the typical class of applications we discuss here, the reference trajectory is the most dominant disturbance. Hence, feedforward control is used to eliminate crosstalk due to residual interaction.

\section{LOW FREQUENCY TRACKING PERFORMANCE}

The objective is to follow a given reference profile at all time instances during the motion. We assume that the plant output $y$ is measured at the location where tracking performance is to be achieved. Therefore, the low frequency tracking problem can be studied considering the transfer function from reference trajectory $r$ to the servo error $e$, Figure 1;

$$
e=S_{o}(s)\left(I-T_{y} G(s) T_{u} F(s)\right) r
$$

with the output sensitivity defined as $S_{o}(s)=(I+$ $\left.T_{y} G(s) T_{u} K(s)\right)^{-1}$. It is common to design a feedforward controller that approximates the inverse of the plant. As many motion systems contain dominant rigid body behavior, it is common practice to use rigid body feedforward inversion by means of acceleration feedforward, so that

$$
F(s)=\tilde{G}_{r b}^{-1} s^{2}
$$

Using the plant model from Equation 4, the transfer function of interest equals

$$
\begin{aligned}
e & =S_{o}(s)\left(I-\left(\tilde{G}_{r b} \frac{1}{s^{2}}+\tilde{G}_{f l e x}\right)\left(\tilde{G}_{r b}^{-1} s^{2}\right)\right) r \\
& =-S_{o}(s) \tilde{G}_{f l e x} \tilde{G}_{r b}^{-1} s^{2} r
\end{aligned}
$$

It is clear that there exists a residual transfer function between the acceleration of the reference trajectory and the servo error. The term $\tilde{G}_{f l e x} \tilde{G}_{r b}^{-1}$ is constant. Hence when no feedback control is applied $\left(S_{o}=I\right)$ the servo error equals the acceleration of the reference profile scaled with the factor $\tilde{G}_{\text {flex }} \tilde{G}_{r b}^{-1}$. When feedback control is applied, the output sensitivity function has at least slope +2 at low frequencies, so that the servo error shows peaks during non-zero jerk phases of a motion, as will be illustrated in Section VII. Note that, as the plant had the same number of inputs as outputs, the spectral radius of this residual is proportional to the dominance measure of Equation 3. Therefore the residual transfer function between acceleration and the servo error is responsible for both cross-talk and low frequency tracking of the diagonal terms. This transfer function can not be reduced using acceleration feedforward. 


\section{MIMO JERK DERIVATIVE FEEDFORWARD}

In the last section it was illustrated that acceleration feedforward has no ability to improve low frequency tracking and reduce cross-talk. In this section the multivariable jerk derivative feedforward will be derived. This feedforward controller is a fourth order Taylor approximate of the inverse plant from Equation 4, so that

$$
\begin{aligned}
F(s) & =\left(\tilde{G}_{r b} \frac{1}{s^{2}}+\tilde{G}_{f l e x}\right)^{-1} \\
& =\left(I+\tilde{G}_{r b}^{-1} \tilde{G}_{f l e x} s^{2}\right)^{-1} \tilde{G}_{r b}^{-1} s^{2} \\
& =\tilde{G}_{r b}^{-1} s^{2}-\tilde{G}_{r b}^{-1} \tilde{G}_{f l e x} \tilde{G}_{r b}^{-1} s^{4}+\mathcal{O}(6)
\end{aligned}
$$

The jerk derivative feedforward controller considers the fourth order approximate so that the new MIMO jerk derivative feedforward controller becomes

$$
\begin{aligned}
F(s)= & F_{a c c} s^{2}+F_{d j e r k} s^{4} \\
& F_{a c c}=\tilde{G}_{r b}^{-1}, F_{d j e r k}=-\tilde{G}_{r b}^{-1} \tilde{G}_{f l e x} \tilde{G}_{r b}^{-1}
\end{aligned}
$$

The first term equals conventional acceleration feedforward while the second term equals (multivariable) jerk derivative feedforward. Note that as rigid body decoupling is applied, only the jerk derivative feedforward part will be nondiagonal.

In order to study the low frequency tracking error, we return to Equation 5. For ease of notation, the situation without feedback control $S_{o}=I$ is studied, the open-loop tracking error then equals

$$
\begin{aligned}
e & =(I-\tilde{G}(s) F(s)) r \\
& =I-\left(\tilde{G}_{r b} \frac{1}{s^{2}}+\tilde{G}_{f l e x}\right)\left(\tilde{G}_{r b}^{-1} s^{2}-\tilde{G}_{r b}^{-1} \tilde{G}_{f l e x} \tilde{G}_{r b}^{-1} s^{4}\right) r \\
& =\tilde{G}_{f l e x} \tilde{G}_{r b}^{-1} \tilde{G}_{f l e x} \tilde{G}_{r b}^{-1} s^{4} r .
\end{aligned}
$$

So there is a residual transfer function between the derivative of jerk and the open-loop servo error. Increasing the order of the approximation in Equation 9 would again lead to a smaller residual. As the residuals will be quadratically smaller each time, choosing higher order feedforward controllers would have limited practical use.

A specific implementation of the feedforward controller is to construct the feedforward controller as a summation of scaled motion phases. The jerk derivative feedforward controller can then be implemented as depicted in Figure 2. Note that due to this implementation, jerk derivative feedforward can be tuned subsequently to tuning acceleration feedforward control. Therefore, manual tuning is facilitated, gradually increasing the complexity of the feedforward controller.

\section{Simulation EXAMPLE}

To illustrate MIMO jerk derivative feedforward, a simulation example of a positioning device is studied. A schematic presentation of the plant is depicted in Figure 3. Two direct drive actuators apply a force in $y$ direction on the beam $B$. The cart $A$ is positioned a distance $x$ from of

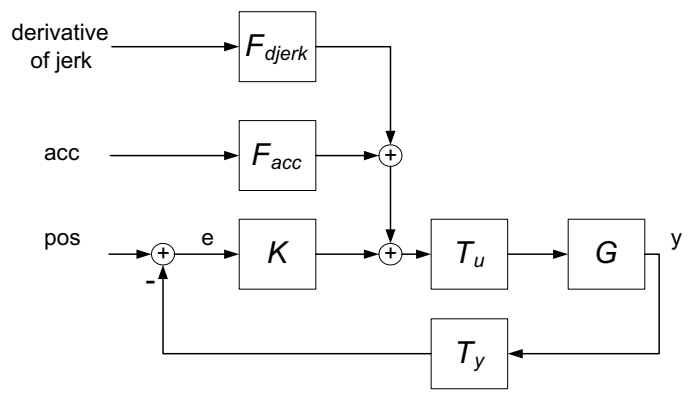

Fig. 2. A suggested implementation of the jerk derivative feedforward controller. Motion phases from the reference trajectory generator are multiplied with constants which can be tuned manually in the time domain.

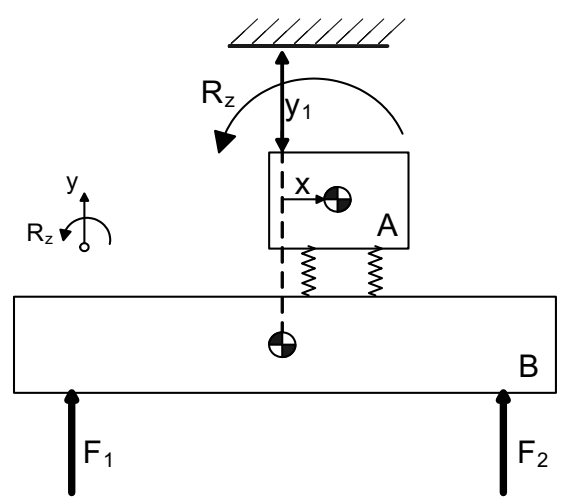

Fig. 3. Schematic picture of a typical positioning multi input multi output device.

the center of gravity of $B$. Two springs model stiffness of the connection between the beam and the cart. The position and rotation of the cart are measured directly as $y_{1}, R_{z}$ respectively. The total mass of the system is $22 \mathrm{~kg}$. The bode diagram of the plant $G(s)$ is depicted in Figure 4.

In order to derive the decoupling transformations, the stiffness between beam and cart is considered to be infinite, hence kinematic relations (distances to and from the center of gravity) can be used to transform the plant,

$$
\left[\begin{array}{c}
y_{1} \\
R_{z}
\end{array}\right]=\underbrace{\left[\begin{array}{cc}
G_{y_{1}, F_{1}}(s) & G_{y_{1}, F_{2}}(s) \\
G_{R_{z}, F_{1}}(s) & G_{R_{z}, F_{2}}(s)
\end{array}\right]}_{G}\left[\begin{array}{c}
F_{1} \\
F_{2}
\end{array}\right]
$$

into a plant which is rigid body decoupled;

$$
\left[\begin{array}{c}
y \\
R_{z}
\end{array}\right]=\underbrace{\left[\begin{array}{cc}
\tilde{G}_{y, F_{y}}(s) & \tilde{G}_{y, T_{R z}}(s) \\
\tilde{G}_{R z, F_{y}}(s) & \tilde{G}_{R_{z}, T_{R}}(s)
\end{array}\right]}_{\tilde{G}=T_{y} G(s) T_{u}}\left[\begin{array}{c}
F_{y} \\
T_{R z}
\end{array}\right] .
$$

The bode diagram of the rigid body decoupled plant, $T_{y} G(s) T_{u}$, is shown in Figure 4. The diagonal dominance measure of Section IV is used to quantify interaction before and after static decoupling with $T_{y}, T_{u}$, Figure 5. Interaction is reduced significantly with rigid body decoupling. As expected, interaction slightly increases around the 

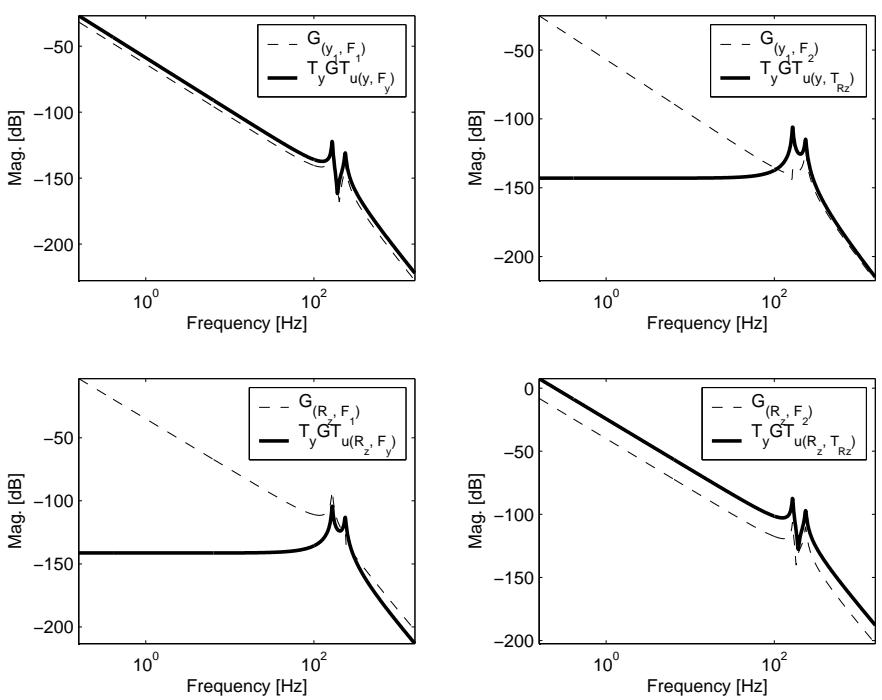

Fig. 4. Bode diagram of the positioning device $G(s)$ and the plant after rigid body decoupling $\tilde{G}=T_{y} G(s) T_{u}$.

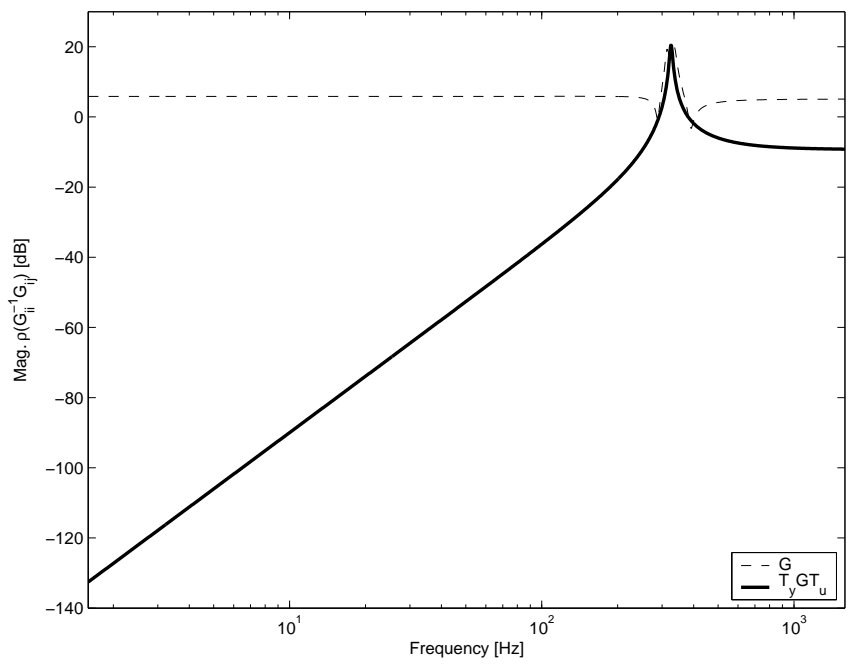

Fig. 5. Diagonal dominance measure of the original plant $G$ and the plant after rigid body decoupling $T_{y} G(s) T_{u}$.

frequencies of the resonance dynamics because the different alignment of the rigid body and the flexible modes. As long as the bandwidth of the feedback controller is below $100 \mathrm{~Hz}$, the system can be considered to be diagonal dominant and the elements of a decentralized feedback controller can be designed independently. Note that in low frequencies, residual interaction in Figure 5 is proportional to $s^{2}$. This indicates that when dynamic decoupling is used to reduce this residual interaction, at least a double differentiating operator is to be included in the feedback loop. This is highly undesired from a practical point of view.

A step in $y$ direction is applied, while feedback control and acceleration feedforward $F(s)=\tilde{G}_{r b}^{-1} s^{2}$ is designed for good tracking of the cart. The rotations of the cart in

\begin{tabular}{|c|c|}
\hline Parameter & Value \\
\hline \hline$x_{\max }$ & $2 \times 10^{-2} \mathrm{~m}$ \\
\hline$v_{\max }$ & $0.8 \mathrm{~m} / \mathrm{s}$ \\
\hline$a_{\max }$ & $15 \mathrm{~m} / \mathrm{s}^{2}$ \\
\hline$j_{\max }$ & $1 \times 10^{3} \mathrm{~m} / \mathrm{s}^{3}$ \\
\hline$d j_{\max }$ & $2 \times 10^{5} \mathrm{~m} / \mathrm{s}^{4}$ \\
\hline
\end{tabular}

TABLE I

REFERENCE TRAJECTORY PARAMETERS.

$R_{z}$ must be kept zero, hence no cross-talk is allowed. The reference profile is of fourth order, generated using the toolbox of [4], with the parameters given in Table I. The various motion phases are plotted in Figure 6. The spectrum of the acceleration of the reference profile is plotted in Figure 7. It is visible that the energy of the reference
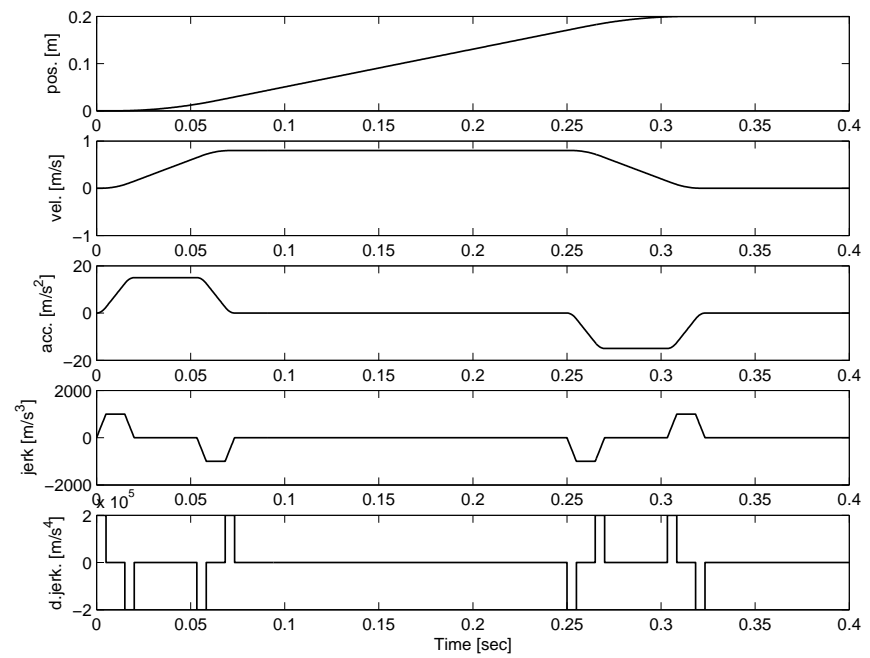

Fig. 6. Motion phases of the reference profile used in the example.

profile is a factor 300 larger in low frequencies than at frequencies around the resonance dynamics. Therefore, mostly low frequency tracking errors can be expected. The servo errors during motion are plotted in Figure 8. Due to residual terms, low frequency tracking errors appear as peaks during jerk phase in $y$ direction. Also, low frequency tracking errors appear in $R_{z}$ direction due to cross-talk (again residual terms).

Next, the MIMO jerk derivative feedforward controller is applied to compensate for these residual terms. Tuning of the parameters of the feedforward controller can be done with hardware in the loop experiments (manually) or considering frequency response functions and using Equation 4. When the same reference profile is applied in $y$ direction, it is visible that the low frequency tracking performance increased spectacularly, bottom Figure 8 (mind the scale). Both low frequency errors in $y$ and cross-talk to $R_{z}$ are eliminated. The remaining servo errors appear due to little residual vibration of the resonance dynamics. In a practical environment, these remaining tracking errors will 


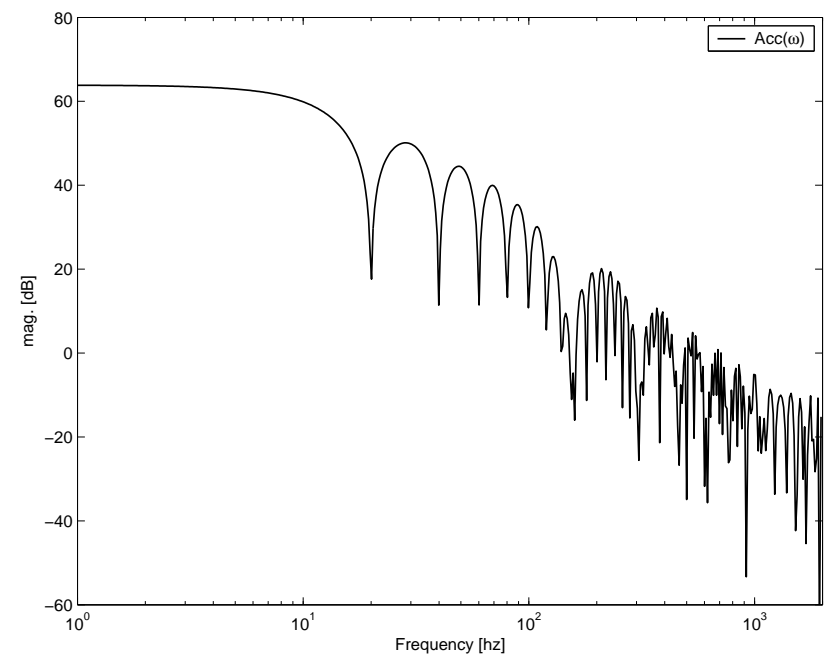

Fig. 7. Spectrum of the acceleration of the reference profile applied in $y$ direction.

not be visible due to the presence of measurement errors and disturbances. Furthermore, we have from Equation 10 that

$$
\left\|\tilde{G}_{\text {flex }} \tilde{G}_{r b}^{-1} \tilde{G}_{\text {flex }} \tilde{G}_{r b}^{-1}\right\|_{2}=2.7 \times 10^{-12}
$$

Which again justifies the fourth order approximation in Equation 8. The plant input in the last phase of the motion is
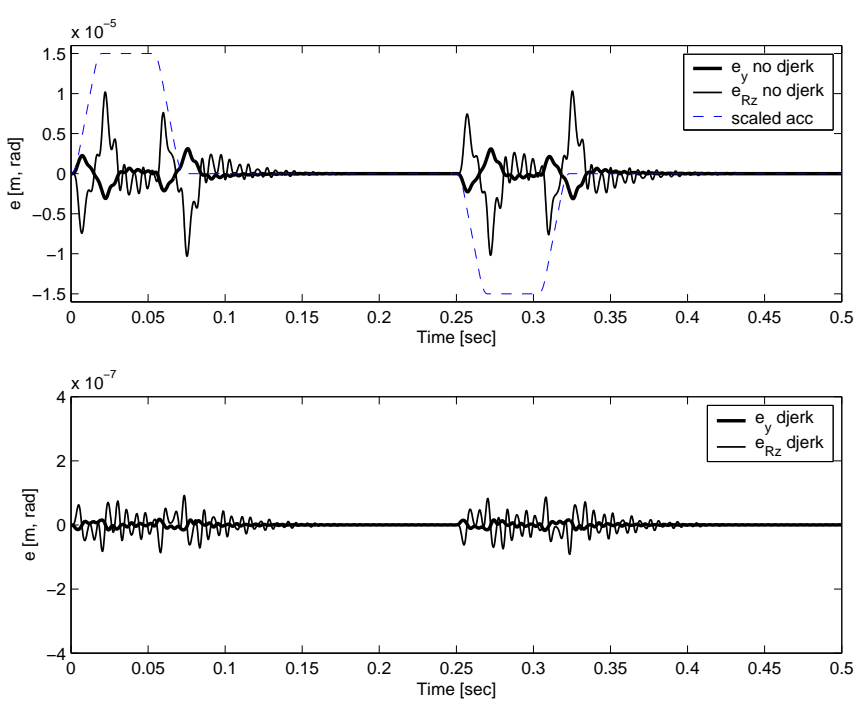

Fig. 8. Servo error of the $y$ and $R_{z}$ axis during step motion in $R_{z}$, see scaled acceleration trajectory in top figure. With standard acceleration (diagonal) feedforward (top figure) and acceleration feedforward with jerk derivative feedforward (bottom figure).

shown in Figure 9. During the acceleration and derivative of jerk phases, the contribution of the feedforward controller is clearly visible, recall Equation 9. In the case without jerk derivative feedforward, the feedback controller tries to compensate for the low frequency tracking errors. As the bandwidth of the feedback controller is limited, performance can not be achieved.
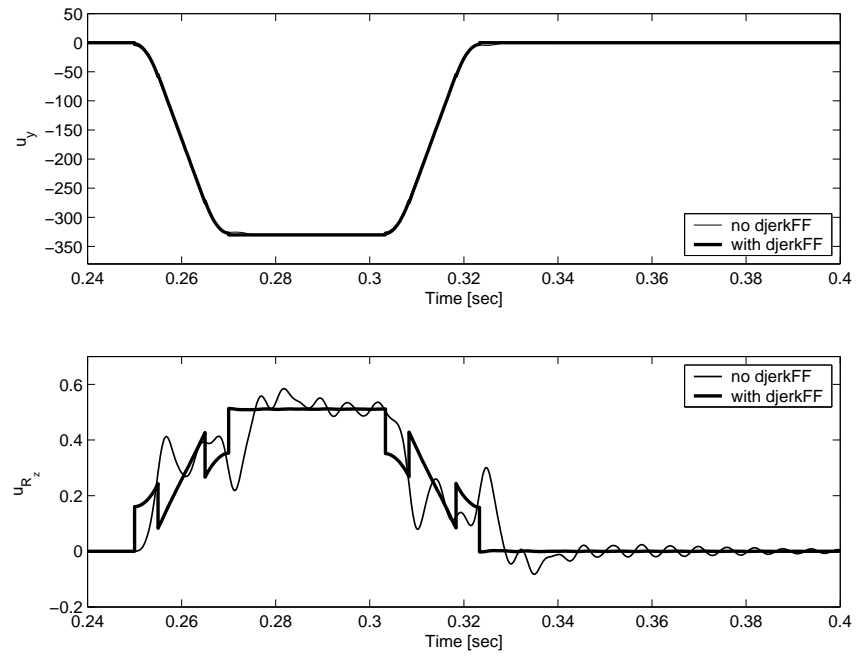

Fig. 9. Plant input in $y$ (top figure) and $R_{z}$ (bottom figure) direction with and without snap feedforward during last phase of the motion.

\section{CONCLUSIONS}

Even when ideal rigid body decoupling and acceleration feedforward are applied, flexible dynamics result in nonzero low frequency residuals. When step and scanning reference profiles are applied, low frequency tracking errors and cross-talk appear. The new multivariable jerk derivative feedforward compensates for these residuals in the low frequency region, thereby significantly increasing tracking performance. It is shown that as the proposed feedforward controller compensates for the summed residuals of all internal flexible modes, the effect of using higher order (than 4) feedforward controllers will result in negligible improvements of performance. Due to the specific structure of the feedforward controller, manual (time domain) tuning is facilitated, providing a straightforward extension of common industrial acceleration feedforward control. Simulations on an example positioning device show promising results.

\section{REFERENCES}

[1] J. Anthonis, H. Ramon, 'Linear mechanical systems and dyadic transfer function matrices', Automatica, vol. 39, 2003, pp 1353-1363.

[2] M. Boerlage, R. Tousain, M. Steinbuch 'Jerk derivative feedforward for motion systems', American Control Conference, vol.5, 2004, pp 4843-4848.

[3] G. Bryant, L. Yeung, 'Multivariable control system design techniques', John Wiley \& Sons, 1996.

[4] P. Lambrechts, M. Boerlage and M. Steinbuch, 'Trajectory planning and feedforward design for electromechanical motion systems', Control Engineering Practice, vol. 13(2), 2005, pp 145-157.

[5] P.H. Meckl, 'Discussion on : Comparison of Filtering Methods for Reducing Residual Vibration', European Journal of Control, vol. 5, 1999, pp 219-221.

[6] P.H. Meckl, P. Arestides, 'Optimized S-Curve Motion Profiles for Minimum Residual Vibration', American Control Conference, vol. 5, 1998, pp 2627-2631.

[7] S. Skogestad, M. Morari, 'Robust Performance of Decentralized Control Systems by Independent Designs', Automatica, vol. 25(1), 1989, pp 199-125.

[8] S. Skogestad, I. Postlethwaite, 'Multivariable feedback control, analysis and design', John Wiley \& Sons, 1996. 
[9] N. Singer, W. Singhose and W.Seering, 'Comparison of Filtering Methods for Reducing Residual Vibration', European Journal of Control, vol. 5, 1999, pp 208-218.

[10] D. Vaes, W. Souverijns, J. De Cuyper, J. Swevers, P. Sasi, 'Optimal decoupling for improved multivariable controller design, applied on an automotive vibration test rig', American Control Conference, vol. 1,2003, pp 785-790.

[11] M. Vandegrift, F. Lewis, 'Improved reduced order model for control of flexible link robots', Conference on Decision and Control, vol. 3, 1993, pp 621-626.

[12] M. van de Wal, G. van Baars, F. Sperling and O. Bosgra, 'Multivariable $H_{\infty}, \mu$ feedback control design for high-precision wafer stage motion', Control Engineering Practice, vol. 10(7), 2002, pp 739-755. 